\title{
Isolated necrosis of central tegmental tracts due to neonatal hypoxic-ischemic encephalopathy: MRI findings
}

\begin{abstract}
Perinatal hypoxia is an old entity that still prevails today and may lead to neurological sequelae that can go unnoticed until a certain age, generating many costs for public health In this case report, we demonstrate on magnetic resonance imaging an unusual pattern of perinatal hypoxia in a preterm 5-month-old infant, involving the central tegmental tracts and briefly discuss its possible pathophysiology.
\end{abstract}

Keywords: magnetic resonance imaging, asphyxia, hypoxic-ischemic encephalopathy, tegmentum, neonates, brainstem

\author{
Volume II Issue I - 202I \\ Tomás de Andrade Lourenção Freddi, Luiz \\ Fellipe Curvêlo Ciraulo Santos, Nelson Paes \\ Fortes Diniz Ferreira, Felipe Diego Gomes \\ Dantas
}

Department of Radiology, Hospital do Coração, Brazil

\begin{abstract}
Correspondence: Tomás de Andrade Lourenção Freddi, Hospital do Coração, 147 Desembargador Eliseu Guilherme Street, São Paulo, SP, 04004-030, Brazil,Tel +55 I 1976059280 Email tomasfredd@gmail.com
\end{abstract}

Received: May 25, 2020 | Published: Febrauary 15, 2021
Abbreviations: MRI, magnetic resonance imaging; HII, hypoxic-ischemic injury; FLAIR, fluid-attenuated inversion recovery; CTT, central tegmental tract; VGB, vigabatrin

\section{Introduction}

Hypoxic-ischemic injury (HII) is one of the most important causes of encephalopathy in neonates, irrespective of gestational age, and may occur in the uterus or during delivery by different intrapartum conditions. In preterm or very low birth weight infants, brain magnetic resonance imaging (MRI) can demonstrate multiple different findings, which a detailed description is beyond the scope of this article, although being periventricular leukomalacia the most frequent (seen in at least $50 \%$ of cases). Brainstem injuries are reported in patients who have suffered from HII and present with symptoms like eating difficulties, speech or language impairment and visual disturbances. ${ }^{1}$

Pre-term patients are often more affected than full-term infants on HII and MRI is suitable to predict the prognosis of these patients and may help in the clinical management. MRI can distinguish between acute and chronic phases of HII, eventually date the time when the patient was affected and also may help with some differential diagnosis like drug toxicity or metabolic diseases. ${ }^{2-4}$ This case report aims to demonstrate a case of CTT involvement due to HII.

\section{Case report}

A 5-month-old female patient, hospitalized for three weeks due to seizures, difficulty swallowing and sucking. There was no history of trauma or previous surgery, and the metabolic screening was negative. She was born prematurely at 35 weeks and her APGAR score was $5 / 7$. MRI showed signal abnormality involving both CTT, suggestive of necrosis.

\section{Discussion}

HII rarely affect the central tegmental tracts (CTT) which are located in the dorsal pons and medulla oblongata, and are compound of ascending and descending fibers, including extrapyramidal axons that connect the red nucleus and the inferior olivary nucleus, being part of the dentato-rubro-olivary system, called Guillain-Mollaret triangle..$^{3-5}$

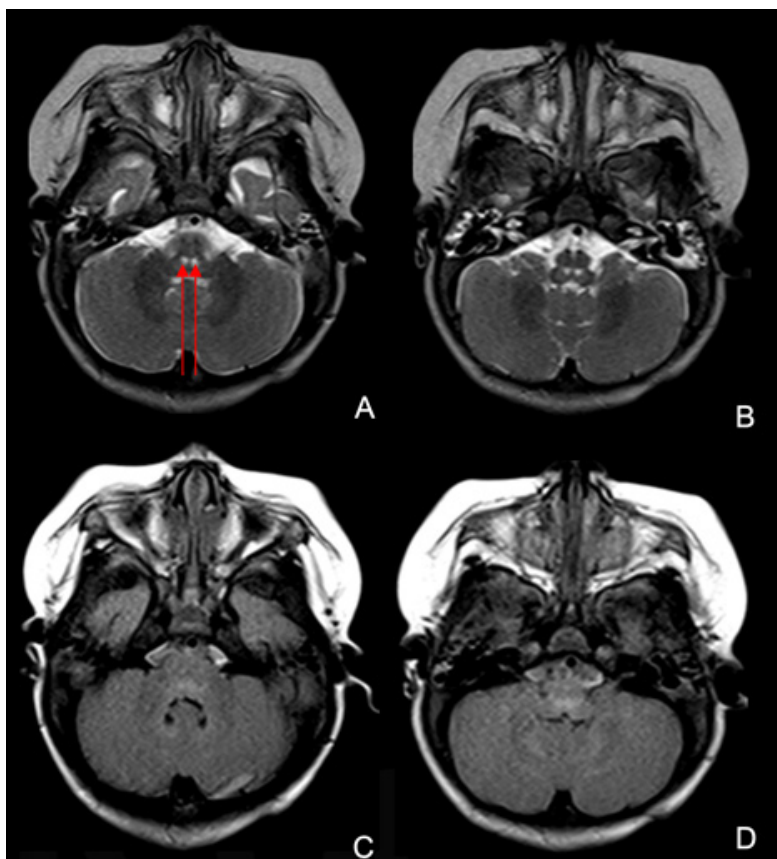

Figure I $(A, B)$ Axial T2-weighted images of the infratentorial compartment show bilateral and symmetrical signal abnormality within the central tegmental tract with mild atrophic effect. (C,D) Axial FLAIR images reveal signal abnormality on the periphery of CTT.

The brainstem tegmentum, including the CTT, is one of the watershed areas of the vertebrobasilar system, supplied by the terminal territories of paramedian and circumferential branches and has a high metabolic demand, factors that may explain its vulnerability to HII. ${ }^{1}$ The CTT is one of the first structures that begin to myelinate in humans, starting at 9 months after conception and being incomplete at 
23 months after birth. ${ }^{2,3}$ CTT is not usually characterized on MRI and hyperintensity in $\mathrm{T} 2$-weighted images and / or diffusion images (DWI) is an unusual neuroimaging finding, ${ }^{5,6}$ described in specific clinical conditions, including HII in preterm infants and Vigabatrin (VGB) toxicity. ${ }^{7}$ Further, it can be seen in patients with metabolic disorders such as nonketotic hyperglycinemia, methionine adenosyltransferase deficiency, leukoencephalopathy with vanishing white matter and 6-pyruvoyltetrahydropterin synthetase deficiency. ${ }^{2-4}$ MRI may improve patients prognosis with clinical support in an earlier disease stage, therefore special attention should be given to brainstem on imaging, ${ }^{8}$ as its involvement in HII is often underdiagnosed. ${ }^{8}$

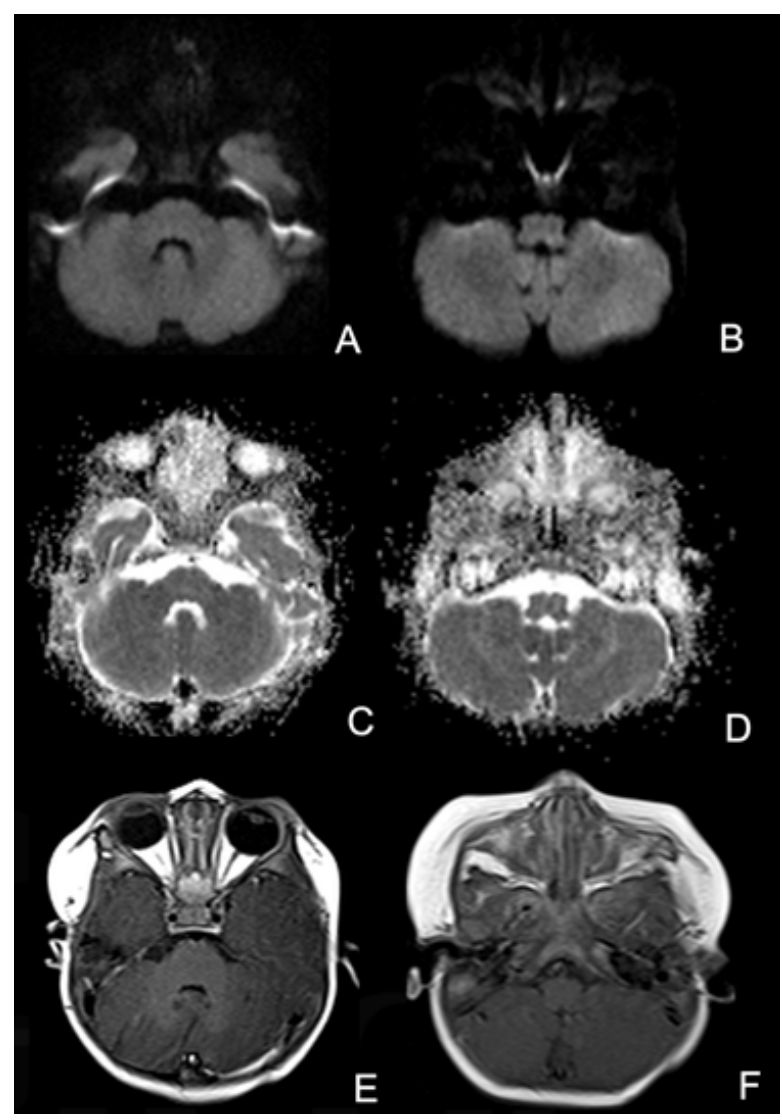

Figure $2(A-D)$ Axial diffusion-weighted images (DWI) and apparent diffusion coefficient (ADC) images do not show acute ischemic lesions. (E,F) Axial TI post contrast images do not reveal contrast enhancement.

\section{Conclusion}

It is crucial to be aware of unusual imaging findings related to HII such as CTT injury, which can be helpful in clinical support. ${ }^{8} \mathrm{~A}$ possible explanation for CTT compromise in HII is their location in a brainstem watershed area, which would make them vulnerable to hypoxia. $^{1}$

\section{Acknowledgments}

None.

\section{Conflicts of interest}

The authors declare no conflicts of interest.

\section{References}

1. Carlo Cosimo Quattrocchi, Giuseppe Fariello, Daniela Longo. Brainstem tegmental lesions in neonates with hypoxic-ischemic encephalopathy: Magnetic resonance diagnosis and clinical outcome. Worl J Radiol. 2016;8(2):117-123.

2. Mallio CA, Van Goethem J, De Bondt T, et al. Symmetrical Central Tegmental Tract Hyperintensity on T2-weighted Images in Pediatrics: A Systematic Review. Annal Behav Neurosci. 2018;1(1):85-93.

3. Shoko Yoshida, Katsumi Hayakawa, Akira Yamamoto, et al. Symmetrical central tegmental tract (CTT) hyperintense lesions on magnetic resonance imaging in children. Eur Radiol. 2009;19(2):462469 .

4. Paramdeep Singh, Amarpreet Kaur, Rupinderjeet Kaur, et al. Symmetrical central tegmental tract hyperintensities on magnetic resonance imaging. J Pediatr Neurosci. 2015;10(3):235-236.

5. Betul Emine Derinkuyua, Evrim Ozmen, HavvaAkmaz-Unlu, et al. A magnetic resonance imaging finding in children with cerebral palsy: Symmetrical central tegmental tract hyperintensity. Brain Dev. 2016;39(3):211-217.

6. Sergio Aguilera-Albesa, Andrea Poretti, Dagmar Honnef, et al. T2 hyperintense signal of the central tegmental tracts in children: disease or normal maturational process?. Paediatric Neuroradiology. 2012;1:3745.

7. Phillip L Pearl, Louis G Vezina, Russell P Saneto, et al. Cerebral MRI abnormalities associated with vigabatrin therapy. Epilepsia. 2009;50(2):184-194.

8. Charlene Mt Robertson, Max Perlman. Follow-up of the term infant after hypoxic-ischemic encephalopathy. Paediatr Child Health. 2006;11(5):278-282. 